\title{
Glioblastoma de la región pineal: reporte de un caso y revisión de la literatura
}

\section{Glioblastoma of the pineal region: case report and review of the literature}

\author{
Ana Arellano A. ${ }^{2}$, Patricio Bustos G. ${ }^{1}$, Melchor Lemp M. ${ }^{1}$, Monserrat Arroyo B. ${ }^{2}$, \\ Francisco Marin $C^{2}{ }^{2}$, Luis Contreras $S^{1}$
}

${ }^{1}$ Neurocirujanos. Departamento de Neurología y Neurocirugía. Hospital Clínico Universidad de Chile.

${ }^{2}$ Residentes Neurocirugía. Departamento de Neurología y Neurocirugía. Hospital Clínico Universidad de Chile.

Instituciones participantes: Hospital Clínico Universidad de Chile.

\section{Resumen}

Los tumores de la región pineal representan el 0,1-0,4\% de los tumores cerebrales primarios 1) Los más frecuentes son los de células germinales, seguidos por los originados de células del parénquima pineal. Menos frecuente, tumores de estirpe glial y misceláneos; 2) De los gliomas, el más frecuente es el astrocitoma bien diferenciado; 3) Los glioblastomas son un hallazgo infrecuente. Reportamos el caso de un paciente de 62 años, cuya clínica se caracteriza por diplopía altitudinal y cefalea. En estudio de imágenes a su ingreso destacan hidrocefalia supratentorial; lesión pineal de aspecto infiltrativo; y aspectos sugerentes de diseminación leptomeningea. En la región espinal se encuentran lesiones metastásicas. Los biomarcadores resultaron negativos. El tratamiento inicial consiste en realizar fenestración de lámina terminalis para manejo de hidrocefalia. Se practica una biopsia de lesión intradural extramedular T10-T11 que es informada como Glioma de alto grado IDH 1 no mutado, con microproliferacion vascular y numerosas figuras mitóticas. Su historia natural permite una sobrevida solo de 5 meses. Las lesiones pineales de estirpe glial son poco frecuentes. Los casos con características de Gliomas Malignos pueden corresponder a Astrocitomas grado 3 OMS y menos frecuentemente a Glioblastomas. Su diagnóstico requiere estudios histológicos y inmunohistologicos detallados, la presencia de diseminación leptomeningea es de mayor frecuencia en los Glioblastomas. Pese a todas las opciones terapéuticas disponibles, este tipo de tumores tienen baja sobrevida.

Palabras clave: Glioblastomas, región pineal.

\section{Abstract}

Tumors of the pineal region represent $0.1-0.4 \%$ of primary brain tumors 1 ) The most frequent are germ cell, followed by originated from pineal parenchyma cells. Less frequently, there are glial and miscellaneous; 2) Among gliomas, the most frequent one is the well-differentiated astrocytoma; 3) The glioblastoma is an infrequent finding. We report a case of 62-yearold patient, with symptoms of altitude diplopia and headache. Imaging studies upon admission revealed supratentorial hydrocephalus; pineal lesion with an infiltrative appearance; and suggestive aspects of leptomeningeal spread. Metastatic lesions were found in the spinal region. The biomarkers were negative. Initial treatment consists of performing fenestration of the lamina terminalis as the management of hydrocephalus. A biopsy of an extramedullary spinal lesion T10-T11 was performed, which was reported as a high-grade IDH 1 non-mutated glioma, with vascular microproliferation and numerous mitotic figures. Natural history allows a survival rate of only 5 months. Pineal glial tumors are rare. Cases with characteristics

\footnotetext{
Correspondencia a:

Ana Arellano Alcántara

Santos Dumont 999, Independencia, Santiago.

Telefono: +56976931260

arellano.alcantara@gmail.com
} 
of Malignant Gliomas may correspond to WHO grade 3 Astrocytomas and less frequently to Glioblastomas. Its diagnosis requires detailed histological and immunohistological studies, the presence of leptomeningeal dissemination is more frequent in Glioblastomas. Despite all the therapeutic options available, these types of tumors have a low survival rate.

Key words: Glioblastomas, pineal region.

\section{Introducción}

Los tumores de la región pineal representan el 0,1-0,4\% de los tumores cerebrales primarios ${ }^{1}$. Dentro de estos, los más frecuentes son los tumores de células germinales (50$75 \%$ ), seguido por los originados de células del parénquima pineal (15-30\%). De forma menos frecuente encontramos aquellos de estirpe glial y tumores misceláneos (meningioma, hemangioblastomas, papiloma del plexo coroideo, metástasis, adenocarcinoma, quimodectomas, linfomas, etc) ${ }^{2}$.

Su forma de presentación más frecuente suele ser la hidrocefalia secundaria a la obstrucción del flujo de LCR desde el tercer ventrículo hacia el acueducto de Silvio. También pueden expresar síntomas por compresión directa de cerebelo, troncoencéfalo o disfunción endocrina.

La etiología glial de alto grado se asocia a un pobre pronóstico y se presenta frecuentemente con diseminación leptomeningea.

Se presenta reporte de un caso y revisión de la literatura cuyo objetivo es presentar a la comunidad neuroquirúrgica sus características clínicas y pronóstico ominoso a pesar de las medidas terapéuticas que actualmente se poseen.

\section{Caso clínico}

Paciente de 62 años, historia de 2 meses de diplopía vertical. Posteriormente se agrega cefalea insidiosa de predominio occipital.

Al examen neurológico destaca Signo de collier (+) bilateral, limitación de la mirada vertical, edema de papila moderado bilateral y afectación parcial bilateral del sexto nervio craneano.

En TAC de cerebro de ingreso (Figura 1) se evidencia masa de aspecto neoplásico en región pineal asociado a hidrocefalia supratentorial. RM confirma lesión de aspecto infiltrativo neoplásico en región pineal.

Ante estos hallazgos, se realiza fenestración de lámina terminalis para manejo de hidrocefalia aguda.

Respecto a marcadores tumorales en sangre y LCR, resultaron negativos (AFP y B-HCG).

Se continúa estudio con RNM de columna total (Figura 2) donde se evidencia lesiones sugerentes de siembra tumoral.

Se realiza biopsia de lesión tumoral intradural extramedular nivel T10-T11 resultando un Glioma de alto grado IDH 1 no mutado, con microproliferación vascular y numerosas figuras mitóticas. Posterior a cirugía, paciente con buena evolución clínica por lo que es dado de alta.

Al mes de evolución, ingresa nuevamente por recurrencia de hidrocefalia, debido a características mixtas de hidrocefalia (obstructiva y trastorno de reabsorción) se decide instalar derivación ventrículo peritoneal. Posteriormente es dado de alta.

A los 8 días el paciente ingresa por compromiso de conciencia. En estudio, destaca crecimiento de lesión pineal con signos de sangrado agudo y vaciamiento ventricular (Figura 4). Se decide limitación del esfuerzo terapéutico. Paciente fallece a los 5 meses desde el diagnóstico.

\section{Discusión}

La glándula pineal es una estructura encapsulada, extra

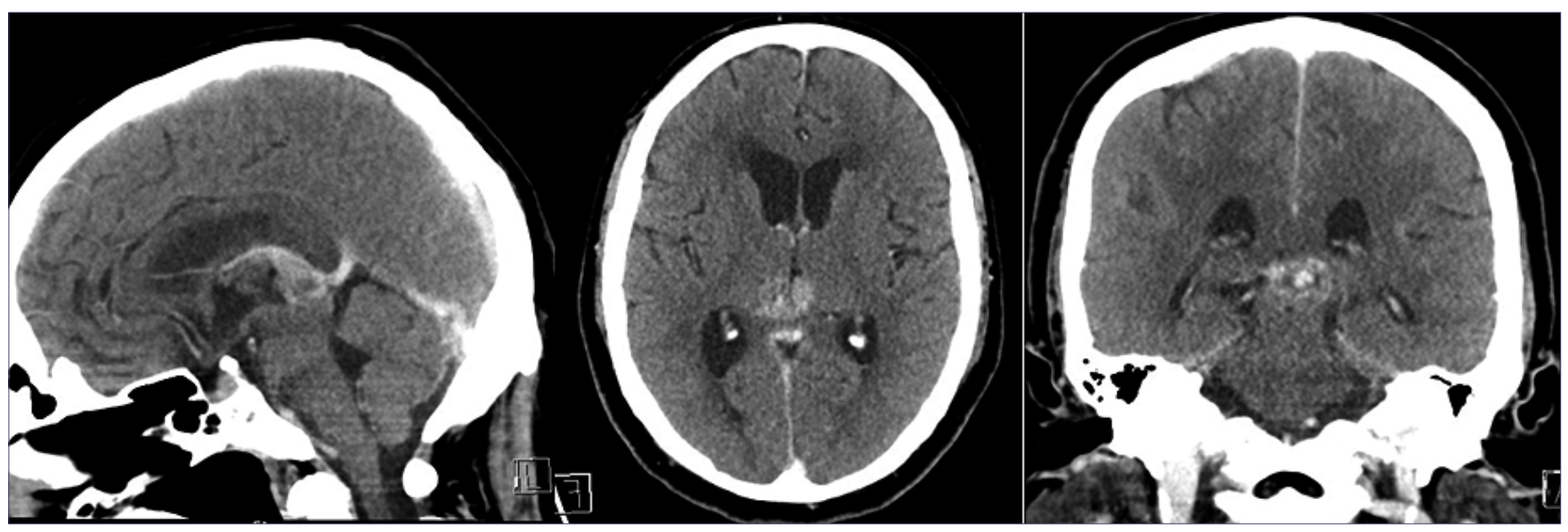

Figura 1. Masa de aspecto neoplásico en región pineal, discretamente hiperdensa, con realce heterogéneo con el contraste, de aprox. 17 x $13 \times 11 \mathrm{~mm}$ asociado a hidrocefalia. 


\section{Reporte de Caso}

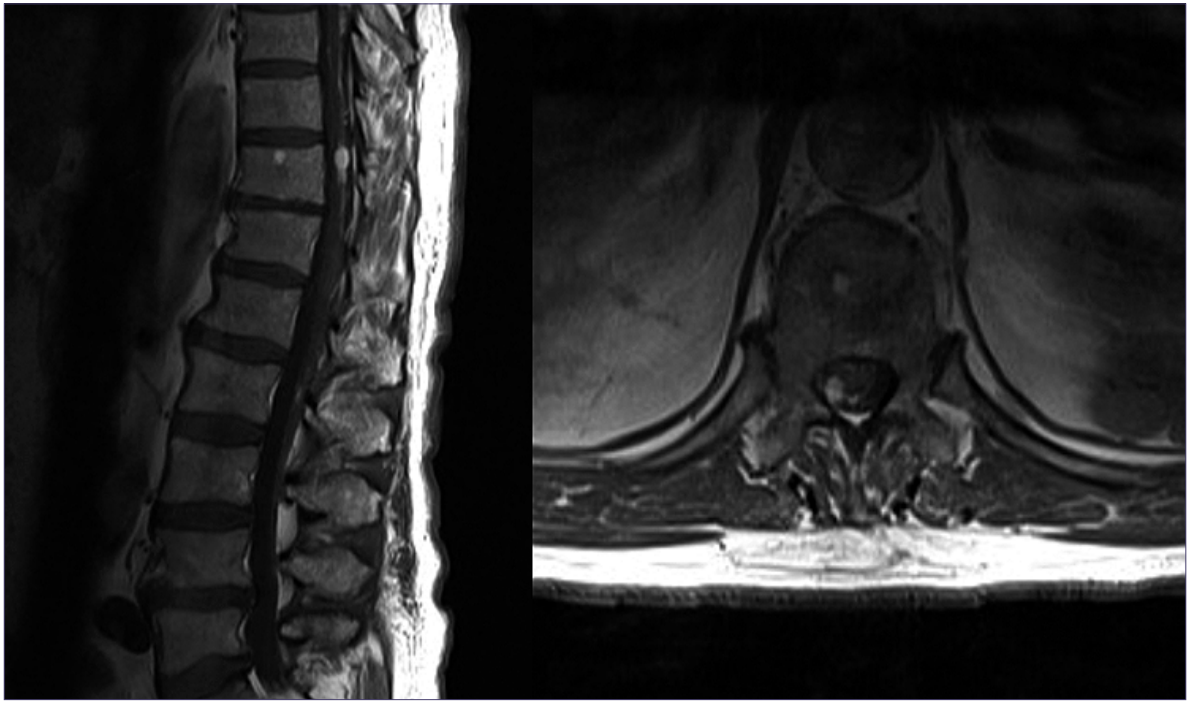

Figura 2. RM columna total T1/Gd. Múltiples lesiones intrarraquídeas, extra e intramedulares que realzan tras el uso de gadolinio. Captación medular leptomeníngea difusa de toda la médula, predominio posterior. En imagen se evidencia lesión a nivel de D11.
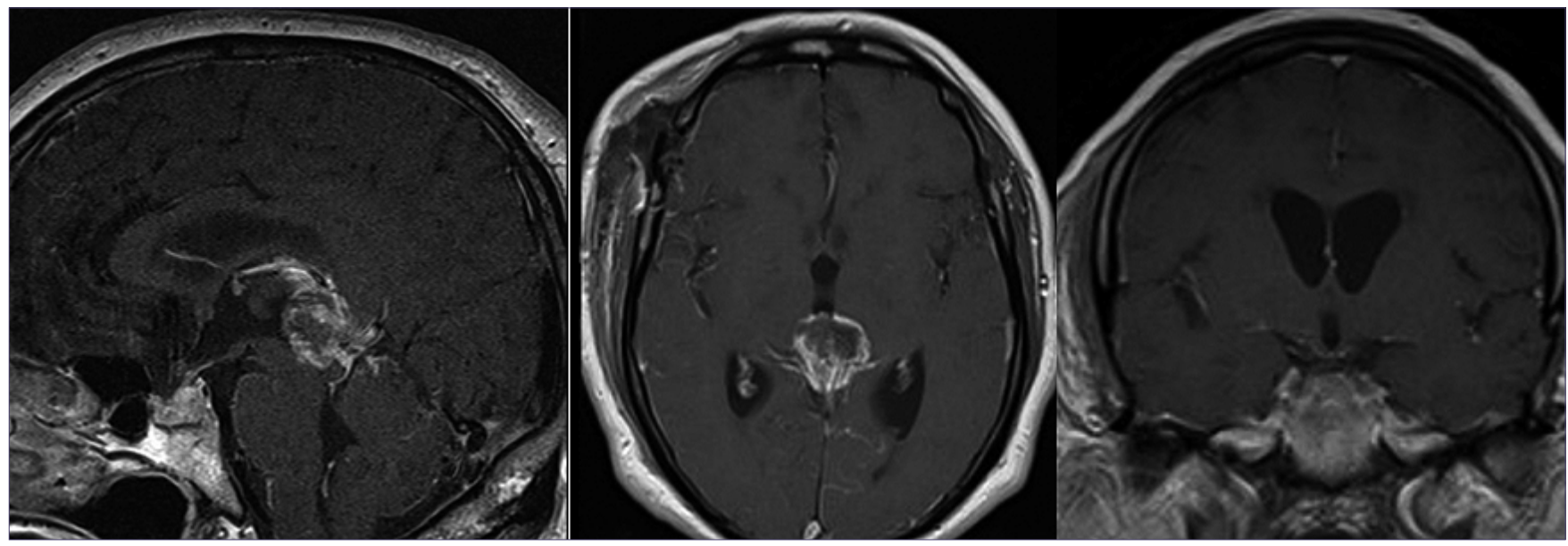

Figura 3. RM cerebro T1/Gd: Proceso expansivo de aspecto neoplásico a nivel de glándula pineal y lámina cuadrigémina con componentes necrótico - quísticos y hemorrágicos de aprox. $28 \times 26 \times 25 \mathrm{~mm}$ con infiltracion hacia ambos tálamos, ejerce efecto de masa en relacion a acueducto. Realce heterogéneo al gadolinio. Realce leptomeningeo en región temporooccipital y parietal.
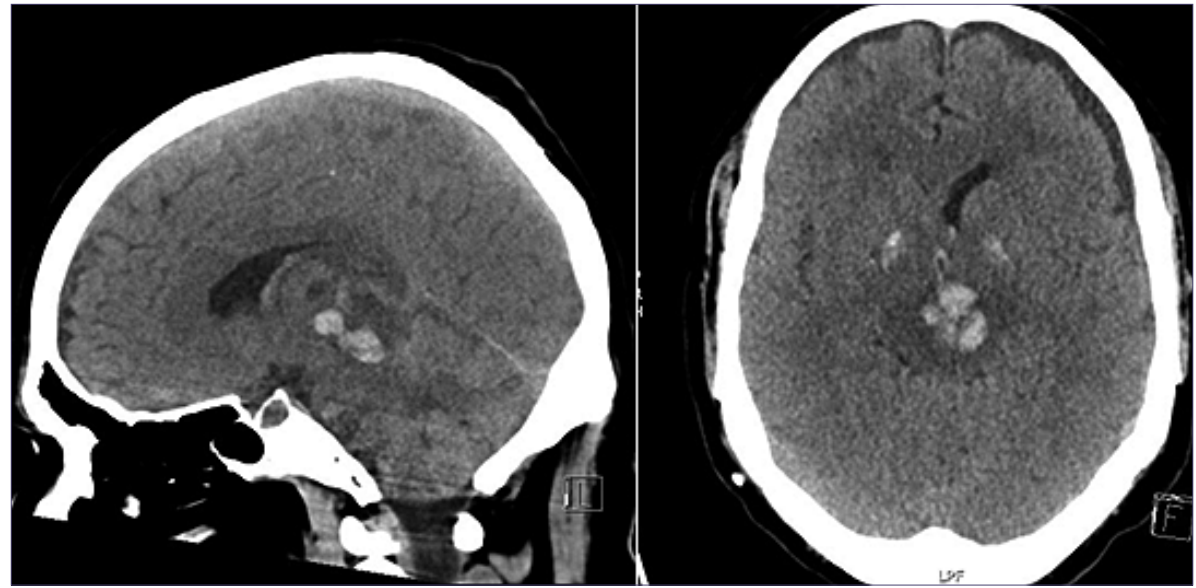

Figura 4. TC de cerebro sin contraste. Lesión expansiva pineal, con signos de sangrado agudo y vaciamiento ventricular. Colecciones yuxtadurales bilaterales sugerentes de higromas. 
axial y que ocupa una depresión entre los tubérculos cuadrigéminos del mesencéfalo.

Está conformada por diversos tipos de células, lo que explicaría la variada histopatología de los tumores de esta región (pinealocitos, astrocitos, celulas endoteliales, tejido conectivo, terminaciones nerviosas, células ependimales de tercer ventrículo).

Dentro de los de estirpe glial, los más frecuentes son los astrocitomas bien diferenciados ${ }^{3}$. En menor medida encontramos astrocitomas anaplásicos, y glioblastomas (GBM), reportándose en la literatura 32 casos, aceptados como glioblastomas incluido el presente (Tabla 1).
Existen 3 formas de presentación clínica más frecuentes; aumento de la presión intracraneana por hidrocefalia (obstrucción del flujo desde el tercer ventrículo al acueducto de Silvio), siendo la cefalea el síntoma inicial más frecuente, sintomatología por compresión directa del cerebelo o troncoencefalo, y menos frecuente como disfunción endocrina.

Respecto al estudio con neuroimágenes, al TC los GBM pineal suelen aparecer como masas con captación de contraste heterogéneo con zonas de baja densidad ${ }^{3}$. Es necesario realizar una RM de cerebro y columna total con y sin contraste antes de cualquier procedimiento para evaluar la extensión de la enfermedad.

\begin{tabular}{|c|c|c|c|c|c|c|c|c|}
\hline \multicolumn{9}{|c|}{ Tabla 1. (RT: radioterapia, QT: quimioterapia) } \\
\hline № & $\begin{array}{c}\text { Año } \\
\text { publicación }\end{array}$ & Estudio & Sexo & Edad & $\begin{array}{l}\text { Hidroce- } \\
\text { falia }\end{array}$ & $\begin{array}{l}\text { Diseminación } \\
\text { leptomeningea }\end{array}$ & Tratamiento & Sobrevida \\
\hline 1 & 1972 & Bradfield et al. & $\mathrm{F}$ & 53 & S/A & No & Resección & $\begin{array}{l}M u \text { e } r t e \\
\text { e } n \text { post } \\
\text { operatorio }\end{array}$ \\
\hline 2 & 1972 & Bradfield et al. & $\mathrm{F}$ & 5 & $S / A$ & No & Shunt & 27 meses \\
\hline 3 & 1973 & DeGirolami et al. & - & $S / A$ & $S / A$ & $S / A$ & Resección + RT & $S / A$ \\
\hline 4 & 1973 & DeGirolami et al. & - & S/A & $S / A$ & $S / A$ & RT & $S / A$ \\
\hline 5 & 1973 & DeGirolami et al. & - & $S / A$ & $S / A$ & $S / A$ & RT & $S / A$ \\
\hline 6 & 1979 & Kalyanaraman & $f$ & 68 & No & $S / A$ & Resección + RT & 4 meses \\
\hline 7 & 1981 & Norbut & M & 36 & $\mathrm{Si}$ & Si (autopsia) & Shunt + RT & 4 meses \\
\hline 8 & 1985 & Frank et al. & $\mathrm{F}$ & 52 & No & $S / A$ & $\begin{array}{l}\text { Biopsia estereotáxica } \\
+ \text { RT }\end{array}$ & 4 meses \\
\hline 9 & 1988 & Edwards et al. & $\mathrm{F}$ & 12 & $\mathrm{~S} / \mathrm{A}$ & $S / A$ & Resección + RT +QT & 18 meses \\
\hline 10 & 1990 & Vaquero et al. & M & 63 & $\mathrm{Si}$ & $S / A$ & $\begin{array}{l}\text { Shunt }+ \text { resección + } \\
\text { whole brain } R T\end{array}$ & 6 meses \\
\hline 11 & 1993 & Pople et al. & $\mathrm{F}$ & 6 & $\mathrm{Si}$ & $\mathrm{Si}(\mathrm{TAC})$ & $\begin{array}{l}\text { Shunt + resección+ } \\
\text { RT local + QT }\end{array}$ & 4 meses \\
\hline 12 & 1998 & Cho et al & $M$ & 63 & No & $S / A$ & Resección + RT & 6 meses \\
\hline 13 & 2003 & Gasparetto et al. & $\mathrm{F}$ & 29 & $\mathrm{Si}$ & No & Shunt + resección & 2 meses \\
\hline 14 & 2005 & Toyooka et al. & M & 49 & $\mathrm{Si}$ & $\begin{array}{l}\mathrm{Si}(\mathrm{RM} \text { de } \\
\text { segumiento) }\end{array}$ & $\begin{array}{l}\text { Shunt + resección + } \\
\text { Qt + RT local }\end{array}$ & 11 meses \\
\hline 15 & 2006 & Amini et al. & $M$ & 40 & $\mathrm{Si}$ & $\mathrm{Si}(\mathrm{RM})$ & $\begin{array}{l}3^{\text {er }} \text { ventriculostomía } \\
\text { + biopsia + resección } \\
+ \text { shunt + RT whole- } \\
\text { brain + Qt }\end{array}$ & 5 meses \\
\hline 16 & 2006 & Amini et al. & M & 43 & $\mathrm{Si}$ & $\begin{array}{l}\mathrm{Si}(\mathrm{Rm} \text { de } \\
\text { seguimiento) }\end{array}$ & $\begin{array}{l}3^{\text {er }} \text { ventriculostomía } \\
+ \text { biopsia + resección } \\
+\mathrm{RT} \text { whole-brain } \\
+\mathrm{Qt}\end{array}$ & 7 meses \\
\hline 17 & 2006 & Amini et al. & $\mathrm{F}$ & 52 & $\mathrm{Si}$ & $\begin{array}{c}\mathrm{Si} \text { (RM de } \\
\text { seguimiento) }\end{array}$ & $\begin{array}{l}3^{\text {er }} \text { ventriculostomía } \\
+ \text { biopsia + RT }\end{array}$ & 2 meses \\
\hline 18 & 2008 & Moon et al. & M & 68 & $\mathrm{Si}$ & $\mathrm{Si}(\mathrm{RM})$ & Resección + shunt & 2 meses \\
\hline 19 & 2013 & Ozgural et al. & $M$ & 60 & $\mathrm{Si}$ & No & $\begin{array}{l}\text { Shunt }+ \text { bx seriadas }+ \\
\text { Rt }+ \text { QT }\end{array}$ & 24 meses \\
\hline 20 & 2014 & Mansour et al. & $M$ & 69 & $\mathrm{Si}$ & No & $\begin{array}{l}3^{\text {er }} \text { ventriculostomía } \\
+ \text { Biopsia + RT + Qt }\end{array}$ & 16 meses \\
\hline 21 & 2014 & Suzuki et al. & M & 65 & $\mathrm{Si}$ & $S / A$ & $\begin{array}{l}\text { Shunt + resección + } \\
R T+Q T\end{array}$ & $S / A$ \\
\hline
\end{tabular}




\begin{tabular}{|c|c|c|c|c|c|c|c|c|}
\hline 22 & 2015 & Matsuda et al. & $\mathrm{F}$ & 31 & $\mathrm{Si}$ & $\mathrm{Si}$ & $\begin{array}{l}\text { Shunt + resección + } \\
\text { RT + QT }\end{array}$ & 5 meses \\
\hline 23 & 2015 & Sugita et al. & $\mathrm{F}$ & 52 & $\mathrm{SI}$ & $S / A$ & $\begin{array}{l}\text { 3er ventriculostomía } \\
\text { +resección+ RT +Qt }\end{array}$ & 24 meses \\
\hline 24 & 2015 & Sugita et al. & M & 18 & $\mathrm{Si}$ & $S / A$ & $\begin{array}{l}\text { Shunt + Resección } \\
\text { parcial + RT + Qt }\end{array}$ & 13 meses \\
\hline 25 & 2015 & Liu et al. & M & 30 & No & $S / A$ & Resección + RT +Qt & $S / A$ \\
\hline 26 & 2016 & Nadvi et al. & M & 19 & $\mathrm{Si}$ & No & $\begin{array}{l}\text { Shunt + Biopsia + } \\
\text { QT + RT }\end{array}$ & $>12$ meses \\
\hline 27 & 2017 & Stowe et al. & M & 65 & No & $S / A$ & Biopsia + Qt + RT & $>38$ meses \\
\hline 28 & 2017 & Orrego et al. & $\mathrm{F}$ & 48 & $\mathrm{Si}$ & No & $\begin{array}{l}\text { Shunt + resección } \\
+\mathrm{RT}\end{array}$ & 12 meses \\
\hline 29 & 2017 & Orrego et al. & M & 50 & $\mathrm{Si}$ & No & $\begin{array}{l}\text { Shunt + resección+ } \\
\text { RT+QT }\end{array}$ & 6 meses \\
\hline 30 & 2017 & Orrego et al. & M & 56 & $\mathrm{Si}$ & $\mathrm{Si}$ & $\begin{array}{l}\text { Shunt + resección + } \\
\text { RT +QT }\end{array}$ & 29 meses \\
\hline 31 & 2017 & Orrego et al. & M & 25 & $\mathrm{Si}$ & No & $\begin{array}{l}\text { Shunt + resección + } \\
\mathrm{RT}+\mathrm{QT}\end{array}$ & 32 meses \\
\hline 32 & 2018 & Caso reportado & M & 62 & $\mathrm{Si}$ & $\mathrm{Si}$ & $\begin{array}{l}3^{\text {er }} \text { ventriculostomia } \\
+ \text { biopsia + Shunt + } \\
\text { RT + QT }\end{array}$ & 5 meses \\
\hline
\end{tabular}

En la literatura se ha descrito infiltración leptomeningea hasta en un $33 \%{ }^{3}$. En nuestra revisión se presentó en 10 de los 32 casos reportados (31\%).

En cuanto a marcadores tumorales, se debe solicitar AFP y BhCG en sangre y/o LCR. La elevación de estos marcadores es patognomónica de tumor de célula germinativa maligno por lo que sería innecesaria la histología.

El manejo debe ser valorado en función de la clínica del paciente; evaluar si presenta signos de hipertensión intracraneana, hidrocefalia, marcadores tumorales, diseminación, estado general del paciente.

En pacientes con hidrocefalia, se aconseja en la literatura resolver por tercerventriculostomía o derivación ventrículo peritoneal en los casos sintomáticos, prefiriendo la primera, permite reducción gradual de la PIC, resolver síntomas antes de la resección tumoral y disminuye las complicaciones como infección, sobredrenaje y siembra peritoneal.

Si hay presencia de síntomas leves y eventual resección total de tumor se puede realizar la instalación de una derivación ventricular externa en el momento de la resección tumoral y en el postoperatorio puede retirarse o instalación de derivación ventrículo peritoneal según necesidad.

En casos asintomáticos y leves, la resección del tumor puede mejorar significativamente el pronóstico.

En nuestra revisión, 65,6\% (21 pacientes) presentó hidrocefalia a lo largo del curso de la enfermedad. De éstos, un $28,5 \%$ se realizó en primera instancia una tercerventrículocisternostomía (vía endoscópica o abierta). De los recién mencionados, solo un $1 / 3$ requirió posteriormente un shunt (Tabla 1).

En general es necesario el diagnóstico histológico, excepto en presencia de marcadores de células germinales malignas. En caso de los glioblastomas, se presentarán tipicamente con alta celularidad, atipia nuclear, actividad mitotica enérgica, proliferación microvascular y/o necrosis.

Si bien aun no es claro el papel de la resección quirúrgica agresiva en casos de glioblastoma pineal, como plantea Orrego et al, se aconseja realizar una resección total en aquellos casos en que se pueda realizar de forma segura. Tanto quimioterapia como radioterapia han demostrado aumentar supervivencia en glioblastomas no pineales ${ }^{5}$, esto podría extrapolarse a los casos de GBM pineal.

En general,los casos de GBM pineal se asocian a un pobre pronóstico, pese a todos los tratamientos disponibles ${ }^{4}$.

En 13 de los 32 casos se optó por manejo con resección tumoral más radioterapia y quimioterapia. En 2 de estos casos no se reportó el tiempo de sobrevida. En los 11 casos restantes se encontró un promedio de sobrevida de 14 meses.

Los pacientes que recibieron radio y quimioterapia sin resección presentan un tiempo de sobrevivencia más alta según lo reportado en la literatura ${ }^{3}$, alcanzando en nuestra revisión un promedio de 19 meses.

En algunos estudios se ha planteado que el intento de resección podría incrementar el riesgo de diseminación leptomeningea, pero aún es tema en debate ${ }^{3}$.

Es importante destacar que un numero no menor de los casos publicados como glioblastoma carecen de una descripción histologica que confirme el fenotipo de este tipo de tumores, en especial necrosis o proliferacion endotelial, si bien el pronóstico con un astrocitoma es muy similar, la diferenciación histológica precisa puede tener importancia en los estudios futuros de este tipo de tumores.

\section{Conclusión}

GBM pineal es una patología poco frecuente y un porcen- 
taje importante de estos casos se presenta con diseminación leptomeningea evidente a través de estudio con RM con gadolinio. El tratamiento se basa, en primera instancia, en tratar la hidrocefalia (forma más común de presentación), seguido de biopsia o resección total o parcial de la lesión, según las condiciones propias del paciente y características de la lesión. Finalmente, radio-quimioterapia. Pese a los tratamientos ofrecidos, estos casos suelen ser de mal pronóstico y baja sobrevida.

\section{Referencias}

1. Hirato J, Nakazato Y. Pathology of pineal region tumors. (2001). J. Neurooncol. 54(3), 239-249

2. Zaazoue, M.A. \& Goumnerova, L.C. Pineal region tumors: a simplified management scheme. Childs Nerv Syst (2016) 32: 2041. https://doi.org/10.1007/s00381-016-3157-4

3. Stowe HB, Miller CR, Wu J, Randazzo DM and Ju AW Pineal Region Glioblastoma, a Case Report and Literatire Review. (2017) Front. <oncol. 7:123. Doi: 10.3389/fonc.2017.00123.

4. Ozgural O, Kahilogullari G, Bozkurt M, Heper AO, Savas A. Primary pineal glioblastoma: a case report. Turk Neurosurg (2013) 23:572-4.10.5137/1019-5149.JTN.5994-12.1

5. Orrego E, Casavilca S, García-Corrochano P, Rojas Meza S, Castillo M, Castaneda C. Glioblastoma of pineal region: report of four cases and literatire review. CNS Oncol (2017). https://doi. org/10.2217/cns-2016-0047

6. Liu, Y., Hao, S., Yu, L., \& Gao, Z. Long-term temozolomide might be an optimal choice for patient with multifocal glioblastoma, especially with deep-seated structure involvement: a case report and literature review. World journal of surgical oncology (2015) 13, 142. doi:10.1186/s12957-015-0558-x

7. Sugita $\mathrm{Y}$, Terasaki M, Tanigawa $\mathrm{K}$, Ohshima $\mathrm{K}$, Morioka $\mathrm{M}, \mathrm{Hi}-$ gaki K, et al. Gliosarcomas arising from the pineal gland region uncommon localization. Neuropathology (2016) 36(1):56-63. doi:10.1111/neup.12226

8. Matsuda, R., Hironaka, Y., Suigimoto, T., \& Nakase, H. Glioblastoma Multiforme in the Pineal Region with Leptomeningeal Dissemination and Lumbar Metastasis. Journal of Korean Neurosurgical Society (2015) 58(5), 479-82.

9. Suzuki R, Suzuki K, Sugiura Y, Takano I, Nagaishi M, Shimizu N, et al. A case of glioblastoma multiforme in the pineal region with intraventricular hemorrhage. No Shinkei Geka (2014) 42(5):42935.

10. Moon KS, Jung S, Jung TY, Kim IY, Lee MC, Lee KH. Primary glioblastoma in the pineal region: a case report and review of the literature. J Med Case Rep (2008) 2:288. doi:10.1186/17521947-2-288

11. Amini A, Schmidit R, Salzman K, Chin S, Couldwell WT. Glioblastoma multiforme of the pineal region. J Neurooncol (2006) 79:307-14. doi:10.1007/ s11060-006-9145-x 